\title{
A Partial Element Equivalent Circuit-metamodel combination for fast tolerance analysis of electromagnetic systems
}

This paper was downloaded from TechRxiv (https://www.techrxiv.org).

\section{LICENSE}

CC BY-NC-SA 4.0

SUBMISSION DATE / POSTED DATE

05-09-2020 / 09-09-2020

\section{CITATION}

Ferranti, Francesco; Antonini, Giulio; Nakhla, Michel (2020): A Partial Element Equivalent Circuit-metamodel combination for fast tolerance analysis of electromagnetic systems. TechRxiv. Preprint. https://doi.org/10.36227/techrxiv.12923117.v1

$\mathrm{DOI}$ 


\title{
A Partial Element Equivalent Circuit-metamodel combination for fast tolerance analysis of electromagnetic systems
}

\author{
Francesco Ferranti $^{*}$, Giulio Antonini**, and Michel Nakhla*** \\ * Institut Mines-Télécom Atlantique, CNRS UMR 6285 Lab-STICC, 29238 Brest CEDEX 3, France (e-mail: \\ francesco.ferranti@imt-atlantique.fr). \\ ** UAq EMC Laboratory, Department of Industrial and Information Engineering and Economics, University of L'Aquila, \\ I-67100 L'Aquila, Italy,(e-mail: giulio.antonini@univaq.it). \\ **** Department of Electronics, Carleton University, Ottawa, ON K1S 5B6, Canada (e-mail: msn@doe.carleton.ca).
}

\begin{abstract}
We propose a combination of the Partial Element Equivalent Circuit method with metamodelling in order to achieve fast tolerance analysis of electromagnetic systems. The proposed model combination can be interpreted as a multifidelity modelling approach. This technique is inspired by the Multilevel Monte Carlo method and provides great benefits in terms of computational resources.
\end{abstract}

\section{Introduction}

Electromagnetic (EM) solvers are fundamental for a variety of important design problems, such as microwave devices, signal and power integrity. The Partial Element Equivalent Circuit (PEEC) method [1] has gained popularity thanks to its ability to represent the electromagnetic behaviour of physical structures by means of circuit elements. As in general for EM solvers, the discretisation and subsequent solution of Maxwell's equations can results in large-scale systems of equations demanding high computational resources. Therefore, the brute force use of EM solvers in design tasks requiring several simulations, such as optimisation and tolerance analysis, becomes infeasible.

Among all the techniques in the literature to speed-up the design flow of complex systems, we focus on multifidelity modelling in this work. The main idea behind multifidelity modelling is to combine models with a different level of accuracy and computational complexity. High accuracy requires high computational resources and vice versa. Several flavours of multifideliy models can be found in the literature [2] with applications in several domains, such as optimisation [3] and tolerance analysis [4].

In the tolerance analysis area, the Monte Carlo (MC) technique is very easy to implement and well-established. One drawback of this technique is the slow convergence that might potentially require a high number of simulations to accurately estimate stochastic information (e.g., mean and variance). Several techniques have been proposed to accelerate the MC analysis, such as Quasi Monte Carlo and Stochastic Collocation techniques [5, 6]. Multifidelity modelling to accelerate MC has been proposed as a multilevel Monte Carlo (MLMC) method in [4]. MLMC has been recently applied to low-frequency EM problems [7]. The EM applications in [7] are examples with a single frequency and the mean value of some quantities of interest is estimated. If a dynamical system is analysed over a frequency range, assuring that the same number and definition of levels is effective for each frequency might be a difficult task. The set of models needs to satisfy some specific conditions about computational complexity and variance in order to achieve an optimal convergence rate [4]. This affects choices such as the number of models (levels) and how to choose the levels. In EM problems, a natural way to define levels is by changing the discretisation mesh size. One additional attractive feature of MLMC is that an error control on the moments estimation is available, as it is the case for MC.

In this work, we propose a simple but very effective multilevel choice for systems that span a broadband frequency range. We select only two models, one high-fidelity model and one low-fidelity model. The high-fidelity model is defined by a PEEC model with a mesh discretisation that ensures high accuracy. This also brings a high computational cost. The low-fidelity model is built based on metamodelling techniques. Metamodels are very efficient and provide a functional relationship between input and output variables [8, 9]. In our case, these variables correspond to physical design parameters and EM quantities of interest (e.g., scattering parameters), respectively. Several basis functions can be used to build this mathematical link, such as radial basis functions, polynomials, and Gaussian processes. The high-fidelity model is kept as a reference for accuracy and used a few times during the tolerance analysis. Instead, the low-fidelity model is taking care of a very high percentage of the needed simulations and it is built in order to well describe the behaviour of the high-fidelity model. We will discuss the estimation of both mean and variance of the EM behavior of interest. Numerical results confirm that the proposed technique allows high speed-ups with respect a standard MC for the same accuracy threshold, while keeping the choice of the levels very simple. 


\section{PEEC method}

The PEEC method [1] applies the Galerkin procedure to Maxwell's equations in Lorentz gauge condition. After the discretisation of materials volumes and surfaces into elementary regions, the Electric Field Integral Equation and continuity equations are used to obtain an EM model that has a circuital description. Considering a transfer function representation, the PEEC method provides a descriptor state-representation. For example, we show below an impedance representation

$$
(s \mathbf{C}(s)+\mathbf{G}(s)) \mathbf{X}(s)=\mathbf{B I}_{p}(s), \mathbf{V}_{p}(s)=\mathbf{B}^{T} \mathbf{X}(s)
$$

where $\mathbf{V}_{p}(s)$ and $\mathbf{I}_{p}(s)$ are the port voltages and currents, and $s$ is the Laplace variable. Admittance and scattering parameters representation are also possible. In the PEEC matrices above, the EM phenomena are represented in terms of resistances, partial inductances and partial capacitances (circuit interpretation of the EM phenomena).

The entries of the partial inductance sub-matrix $\mathbf{L} p(s)$ and potential coefficient sub-matrix $\mathbf{P}(s)$ in the block-structure of the matrix $\mathbf{C}(s)$ are defined as follows

$$
\begin{aligned}
& L p_{n, m}(s)=\mu \int_{V_{n}} \int_{V_{m}} g\left(\mathbf{r}, \mathbf{r}^{\prime}, s\right) d V_{m} d V_{n} \\
& P_{n, m}(s)=\frac{1}{\varepsilon} \int_{S_{n}} \int_{S_{m}} g\left(\mathbf{r}, \mathbf{r}^{\prime}, s\right) d S_{m} d S_{n} .
\end{aligned}
$$

using the scalar Green's functions with retardation of the type

$$
g\left(\mathbf{r}, \mathbf{r}^{\prime}, s\right)=\frac{1}{4 \pi} \frac{e^{-s \tau}}{\left|\mathbf{r}-\mathbf{r}^{\prime}\right|}
$$

with the delay equal to $\tau=\frac{\left|\mathbf{r}-\mathbf{r}^{\prime}\right|}{c_{0}}$ and $c_{0}$ equal to the speed of light in vacuum. The reader can refer to [1] for more details about the PEEC method. Several flavours of the PEEC method exists depending on how the exponential function in the integrals above is treated. For this work, we used the so called center-to-center approximation:

$$
L p_{n, m}^{(c c)}(s)=e^{-s \frac{R_{n, m}}{c_{0}}} L p_{n, m}^{(q s)}, P_{n, m}^{(c c)}(s)=e^{-s \frac{R_{n, m}}{c_{0}}} P_{n, m}^{(q s)}
$$

where $R_{n, m}$ is the Euclidean distance between the centers of the mesh cells $n$ and $m$. The delay center-to-center between each couple of mesh cells is used to approximate the delay of the interaction of each mesh cell couple and the exponential term is taken out of the integrals. This PEEC formulation has been proven accurate for several application cases and less computationally expensive than the PEEC solution where the integrals are kept into the integrals when computing the partial inductances and potential coefficients terms. This formulation is also more accurate than the so-called quasi-static (qs) case where the exponential terms are just fixed equal to one.

\section{$3 \quad$ MC and Multifidelity modelling}

In the design of a system of interest, design parameters such as layout features influence the system behaviour and therefore the design performances. Design tasks, such as optimisation and tolerance analysis, require multiple simulations where the design parameters are varied. Let us consider $d$ design parameters as $\boldsymbol{\xi}=\left[\xi_{1}, \ldots, \xi_{d}\right] \in \mathbb{R}^{d}$, then we can introduce the influence of these parameters into the state-space PEEC equations (1) as

$$
(s \mathbf{C}(s, \xi)+\mathbf{G}(s, \boldsymbol{\xi})) \mathbf{X}(s, \boldsymbol{\xi})=\mathbf{B I}_{p}(s), \mathbf{V}_{p}(s, \boldsymbol{\xi})=\mathbf{B}^{T} \mathbf{X}(s, \boldsymbol{\xi})
$$

Our contribution targets tolerance analysis and the estimation of stochastic quantities, such as mean and variance, of some EM quantities of interest that depend on random variations of the $\xi$ parameters. We estimate stochastic moments of the scattering parameters $\mathbf{S}(s, \boldsymbol{\xi})$ in this work. The formulas in what follows are considered for a scalar function for ease of notation. The extension towards vectors and matrices is straightforward considering element-wise operations. The $m$-th raw and central stochastic moment of $S(s, \boldsymbol{\xi})$ (without loss of generality, we assume here continuous random variables) can be expressed as

$$
\mathbb{E}\left[Q^{m}(s, \boldsymbol{\xi})\right]=\int_{\boldsymbol{\Gamma}} Q^{m}(s, \boldsymbol{\xi}) W(\boldsymbol{\xi}) d \boldsymbol{\xi}
$$

with $m \in \mathbb{N}$, where $\mathbb{E}[\cdot]$ represents the expectation operation, the $Q(s, \boldsymbol{\xi})$ quantity can denote $S(s, \boldsymbol{\xi})$ or $S(s, \boldsymbol{\xi})-$ $E[S(s, \boldsymbol{\xi})]$, for raw and central moments, respectively. We call here $\mu_{m}^{\prime}(Q)$ and $\mu_{m}(Q)$ the $m$-th raw and central moments, respectively. $W(\boldsymbol{\xi})$ is a joint probability density function.

In this work, we use the following assumption: although the exact EM behaviour is not perfectly known due to Maxwell's equations discretisation in EM solvers, the common way to use EM solvers in engineering to get accurate results is by choosing settings (e.g., a fine mesh discretisation) that can provide a high accuracy. Therefore, we assume that the response $S(s, \boldsymbol{\xi})$ provided by the PEEC solver is the correct EM response. An estimation of the moments above described can be obtained by the MC approach, where a set of random samples based on the joint probability density function of $\boldsymbol{\xi}$ is used. After the function $S(s, \boldsymbol{\xi})$ is sampled in the $\boldsymbol{\xi}$ space, the collected data can be used to 
estimate stochastic moments by sample-based formulas. It is important to use estimators that are unbiased and have minimal variance compared with other unbiased estimators. In this perspective, for example the mean $\mathbb{E}[S(s, \boldsymbol{\xi})]$ and variance $\mathbb{E}\left[(S(s, \boldsymbol{\xi})-E[S(s, \boldsymbol{\xi})])^{2}\right]$ can be estimated using $R$ independent and identically distributed (i.i.d.) samples $S_{R}:=\left(S\left(s, \boldsymbol{\xi}_{r}\right)\right)_{r=1, \ldots, R}$ as

$$
\begin{array}{r}
\mathbb{E}[S(s, \boldsymbol{\xi})] \approx m_{1}\left(S_{R}(s, \boldsymbol{\xi})\right)=\sum_{r=1}^{R} \frac{S\left(s, \boldsymbol{\xi}_{r}\right)}{R} \\
\mathbb{E}\left[(S(s, \boldsymbol{\xi})-E[S(s, \boldsymbol{\xi})])^{2}\right] \approx h_{2}\left(S_{R}(s, \boldsymbol{\xi})\right)=\frac{R T_{2}-T_{1}^{2}}{(R-1) R}
\end{array}
$$

Both (8a)-(8b) represent an unbiased estimator of the corresponding moments [10]. For the estimation of the second central moment (the variance), we use the so-called $h$-statistics that provides an unbiased estimator with minimal variance with respect to other unbiased estimators [10]. Based on the samples $S\left(s, \boldsymbol{\xi}_{r}\right), r=1, \ldots, R, T_{a}$ in $h$-statistics is defined as a power sum $T_{a}=\sum_{r=1}^{R} S^{a}\left(s, \boldsymbol{\xi}_{r}\right)$ with $a \in \mathbb{N}$. Generalisation of (8a)-(8b) for higher order moments can be found in $[10,11]$.

In this work, we consider mean and variance as the stochastic moments of interest. The described estimators are based on random sampling. Their mean square error $(M S E)$ can be defined in general as a bias term squared and a variance term (we omit $(s, \boldsymbol{\xi})$ into $S(s, \boldsymbol{\xi})$ and $S_{R}(s, \boldsymbol{\xi})$ for ease of notation in what follows). In the case of an unbiased estimator, such as $m_{1}$ and $h_{2}$, only the variance term will appear [11]

$$
\begin{aligned}
& M S E\left(m_{1}\left(S_{R}\right)\right)=\mathbb{E}\left[\left(m_{1}\left(S_{R}\right)-\mu_{1}^{\prime}(S)\right)^{2}\right]=\operatorname{Var}\left(m_{1}\left(S_{R}\right)\right) \\
& M S E\left(h_{2}\left(S_{R}\right)\right)=\mathbb{E}\left[\left(h_{2}\left(S_{R}\right)-\mu_{2}(S)\right)^{2}\right]=\operatorname{Var}\left(h_{2}\left(S_{R}\right)\right) \\
& \operatorname{Var}\left(m_{1}\left(S_{R}\right)\right)=\frac{\mu_{2}(S)}{R}, \operatorname{Var}\left(h_{2}\left(S_{R}\right)\right)=\frac{\mu_{4}(S)}{R}-\frac{\mu_{2}^{2}(S)(R-3)}{(R-1) R}
\end{aligned}
$$

Using $h_{2}\left(S_{R}\right)$ to estimate $\mu_{2}(S)$ provides an unbiased estimator of $\operatorname{Var}\left(m_{1}\left(S_{R}\right)\right)$. For this formula, estimating the needed samples $R$ to achieve a certain target $M S E$ is straightforward. An unbiased estimator for $\operatorname{Var}\left(h_{2}\left(S_{R}\right)\right)$ is discussed in [11]. Both variance terms in $(9 \mathrm{c})$ are of order $\mathcal{O}\left(R^{-1}\right)$, which allows reformulating $\operatorname{Var}\left(h_{2}\left(S_{R}\right)\right)$ in a more suitable form in order to estimate the needed samples $R$ to achieve a certain target $M S E$ [11]. The maximum number of samples between the two estimations is then chosen. We note that MC and the proposed multifidelity approach start from an initial number of samples, the needed number of samples to achieve the chosen $M S E$ value is estimated, then new simulations are performed and the loop continues till the chosen $M S E$ value is achieved.

The MLMC technique generalises the MC technique. A set of models is combined in a specific form. Let us consider $L+1$ levels and therefore $L+1$ models that provide $\left\{S_{l}(s, \boldsymbol{\xi})\right\}_{l=0}^{L}$ as corresponding output. We assume the $L$-th model is the most accurate and computationally expensive, while the 0 -th model is the least accurate and computationally expensive in the set of $L+1$ levels. We assume the $L$-th model gives what we consider the exact EM behaviour $S_{L}=S$ and this model is also used by the MC analysis. The estimation of mean and variance of $S(s, \boldsymbol{\xi})$ can be obtained as described in [4] and [11], respectively

$$
\begin{gathered}
\mathbb{E}[S(s, \boldsymbol{\xi})] \approx m_{1, M L M C}\left(\left\{S_{l, R_{l}}\right\}_{l=0}^{L}\right)=m_{1}\left(S_{0, R_{0}}\right)+\sum_{l=1}^{L} m_{1}\left(S_{l, R_{l}}-S_{l-1, R_{l}}\right) \\
\mathbb{E}\left[(S(s, \boldsymbol{\xi})-E[S(s, \boldsymbol{\xi})])^{2}\right] \approx h_{2, M L M C}\left(\left\{S_{l, R_{l}}\right\}_{l=0}^{L}\right)=h_{2}\left(S_{0, R_{0}}\right)+\sum_{l=1}^{L}\left(h_{2}\left(S_{l, R_{l}}\right)-h_{2}\left(S_{l-1, R_{l}}\right)\right)
\end{gathered}
$$

Considering the assumption $S_{L}=S$, both estimators $m_{1, M L M C}$ and $h_{2, M L M C}$ are unbiased. Concerning their $M S E$ (thus their variance), we assume to use i.i.d. samples to sample and evaluate $S_{0}(s, \boldsymbol{\xi})$ and each pair $\left[S_{l}(s, \boldsymbol{\xi}), S_{l-1}(s, \boldsymbol{\xi})\right]$. The following variance formula for the $m_{1, M L M C}$ estimator can be obtained [4]

$$
\operatorname{Var}\left(m_{1, M L M C}\right)=\operatorname{Var}\left(m_{1}\left(S_{0, R_{0}}\right)\right)+\sum_{l=1}^{L} \operatorname{Var}\left(m_{1}\left(S_{l, R_{l}}-S_{l-1, R_{l}}\right)\right)
$$

A similar formula can be obtained for $h_{2, M L M C}$ as a sum of variance contributions. However, the formula is more involved and we refer to [11] for the details.

For a chosen $M S E$ value related to the computation of mean and variance of $S(s, \boldsymbol{\xi})$, the number of samples $\left\{R_{l}\right\}_{l=0}^{L}$ needed to achieve this error level and minimize the total computational cost of the tolerance analysis can be found by simple analytical formulas obtained after a constrained minimisation problem [4, 11]. Therefore, as in (9c), the needed number of samples can be easily computed [4, 11].

Choosing the number and definition of levels might not be a simple choice, especially for a broadband design of interest. These choices strongly influence the speed-up that MLMC can achieve over MC.

What we propose is to reduce the number of levels to only two levels. This removes in principle some flexibility, but it provides a very simple and effective choice. Therefore, we are in the scenario of one high-fidelity model (HFM) and one low-fidelity model (LFM). The LFM is generated by using metamodelling techniques and it accurately approximates 
the HFM. Therefore, we consider a metamodel as $S_{\text {metamodel }}(s, \boldsymbol{\xi}) \approx S_{L}(s, \boldsymbol{\xi})$. In this work, the LFM is created by using a latin hypercube sampling (LHS) approach and radial basis functions (RBF) and polynomial functions for the metamodel construction. The LHS collects the data $S_{L}\left(s, \boldsymbol{\xi}_{q}\right), q=1, \ldots, Q$ and the metamodel is then built

$$
S_{\text {metamodel }}(s, \boldsymbol{\xi})=\sum_{q=1}^{Q} w_{q}(s) \phi\left(\boldsymbol{\xi}-\boldsymbol{\xi}_{q}\right)+\sum_{b=1}^{B} w_{b}(s) p_{b}(\boldsymbol{\xi})
$$

Different radial basis functions $\phi\left(\boldsymbol{\xi}-\boldsymbol{\xi}_{q}\right)$ exist in the literature. We selected as an example the multiquadrics basis functions $\phi\left(\boldsymbol{\xi}-\boldsymbol{\xi}_{q}\right)=\sqrt{r_{q}^{2}+c^{2}}$ where $r_{q}$ represents the euclidean distance between $\boldsymbol{\xi}$ and $\boldsymbol{\xi}_{q}$ and c is a positive parameter. Other choices are possible. The $B$ polynomial functions were chosen to be $\left[1, \xi^{(1)}, \xi^{(2)}, \ldots, \xi^{(d)}\right]$.

The HFM (level 1) is kept as a reference for accuracy and called a few times (this means a few samples) during the tolerance analysis. Instead, the LFM (level 0) takes care of a very high percentage of the needed simulations and it accurately approximates the behaviour of the HFM. In this way, their difference (level 1 - level 0 ) will be low and a few samples will be called by this difference term in the related MLMC formulas. We note that it might be tempting to use only the LFM. However, this will introduce a bias term into the $M S E$ formulas, which might be difficult to compute accurately. We note that having a more or less accurate LFM will bring less or more samples for the difference term (level 1 - level 0) into the MLMC formulas. The generation of a metamodel can be automated by adaptive sampling and modelling techniques. However, this is beyond of the scope of this work.

\section{Numerical results}

A finger capacitor was used as a validation example. Fig. 1 shows the layout of the structure. The thickness of conductors and dielectrics is equal to $100 \mu \mathrm{m}$ and $840 \mu \mathrm{m}$, respectively; $\mathrm{WP}=300 \mu \mathrm{m}$, WC $=400 \mu \mathrm{m}$, WR $=700$ $\mu \mathrm{m}$. The stochastic parameters are uniform random variables varying in the $\pm 10 \%$ range with central values $\mathrm{L}=1400$ $\mu \mathrm{m}, \mathrm{W}=600 \mu \mathrm{m}, \mathrm{G}=200 \mu \mathrm{m}, \mathrm{LP}=950 \mu \mathrm{m}$ and $\mathrm{GR}=650 \mu \mathrm{m}$. The electrical port of interest is defined by the blue dots. The metal is copper, while the substrate is made by a dielectric with relative permittivity of 6.5 . The frequency range of interest is $[1-7] \mathrm{GHz}$ and it was sampled by 51 samples. Each frequency sample is considered separately for the tolerance analysis. The $5 \mathrm{D}$ space of the stochastic parameters is composed of $\boldsymbol{\xi}=[L, W, G, L P, G R]$. This space was sampled by a MC technique and the proposed technique. The HFM is a PEEC model with a mesh resolution $\lambda_{\text {min }} / 40$ ( $\lambda$ is the wavelength). A higher mesh resolution leads to the same accuracy of the EM results (and therefore to an unnecessary higher computational complexity), while a lower mesh resolution leads to accuracy losses. This is the only model used by the MC approach. The EM quantity of interest is the scattering parameter $S_{11}(s=j 2 \pi f r e q u e n c y, \boldsymbol{\xi})$.

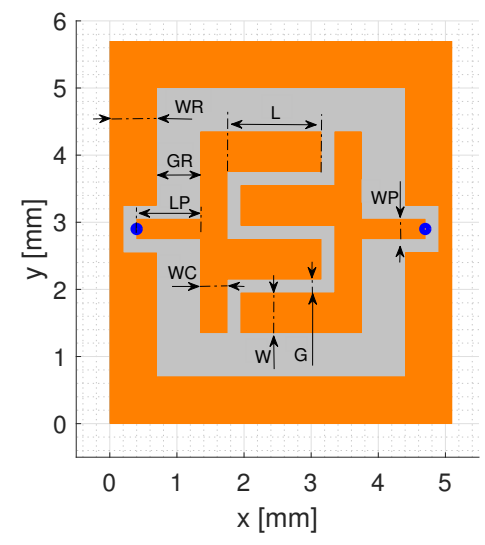

Figure 1: Layout of the finger capacitor.

The average CPU time needed by the HFM model for one frequency sample simulation is $178 \mathrm{~s}$, while the same CPU time quantity for the metamodel is equal to $1 \mathrm{~ms}$. The LHS sampling to build the metamodel $S_{\text {metamodel }}(j 2 \pi f r e q u e n c y, \boldsymbol{\xi})$ used 50 samples in the $\boldsymbol{\xi}$ space. We consider an $M S E$ equal to $10^{-6}$ as accuracy objective for the estimators of mean and variance of the real and imaginary part of $S_{11}$. Fig. 2 shows the speed-up obtained by the proposed technique with respect to a $\mathrm{MC}$ analysis, when both techniques target the same $M S E$ value. This speed-up is calculated considering for each frequency sample: a) for the MC, the CPU time needed to run the MC using the HFM; b) for the proposed technique, the CPU time needed to collect the $S_{11}$ data samples related to the LHS sampling, to build the metamodel and to run the MLMC analysis with the two levels (LFM and HFM). The needed number of samples to run these two techniques is chosen by (9c) for $\mathrm{MC}$ and by the formulas in $[4,11]$ for the proposed two-level multifidelity technique. Both techniques were initialised with $50 \boldsymbol{\xi}$ random samples for each level involved (the HFM level only in MC, the LFM and HFM levels in the multifidelity scheme). Fig. 3 compares the mean and variance estimation of the real and 
imaginary part of $S_{11}$ for the MC analysis and proposed technique. As clearly demonstrated by the results, the proposed multifidelity scheme achieves a significant speed-up ensuring the same $M S E$ of a MC analysis.

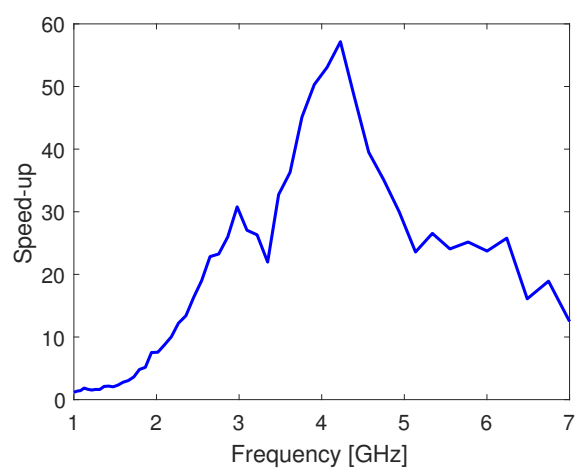

Figure 2: Speed-up of the proposed technique with respect to a MC analysis.
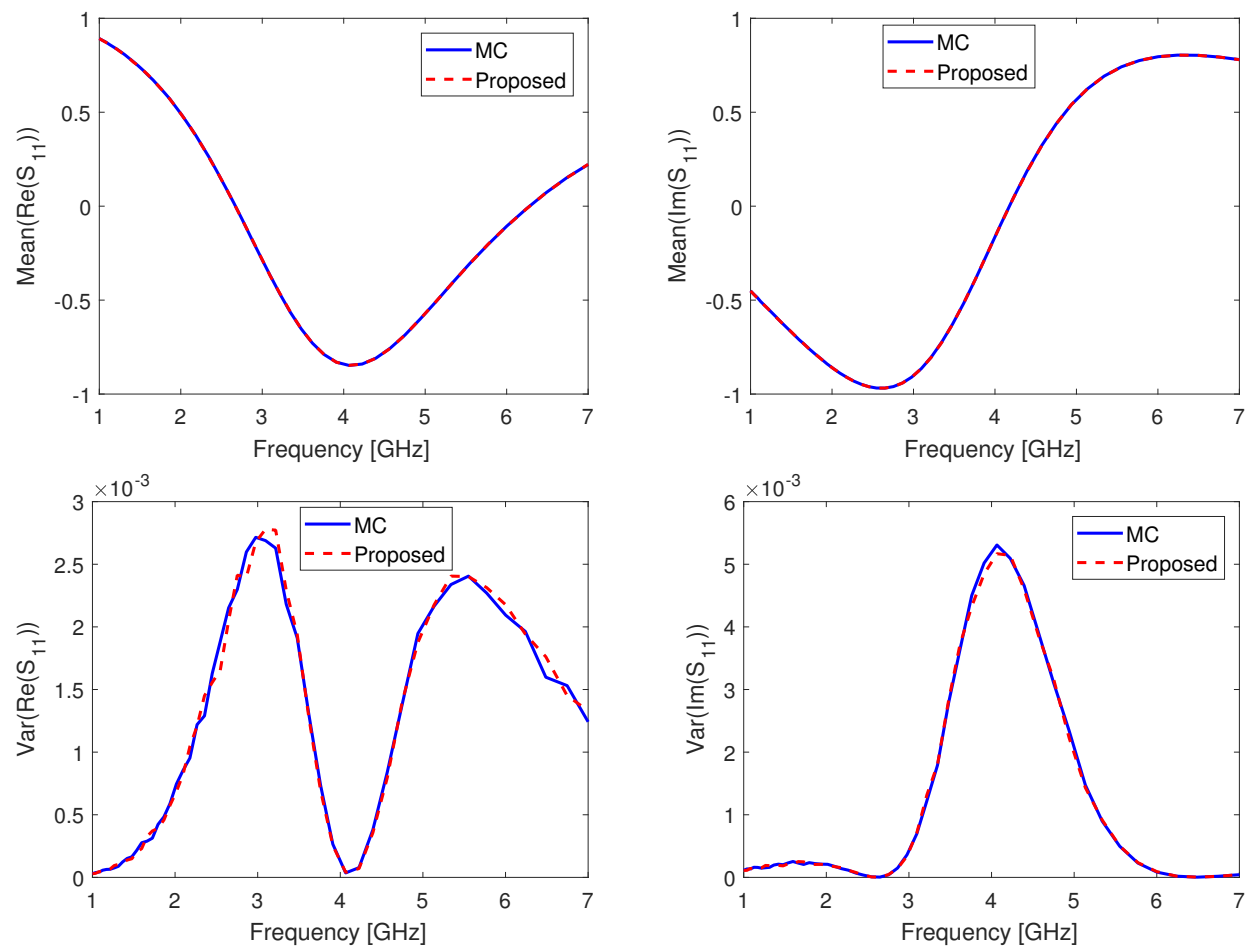

Figure 3: Mean and variance of the real and imaginary part of $S_{11}$.

\section{Conclusion}

A two-level multifidelity method has been proposed for fast tolerance analysis of EM systems that span a broadband frequency range. The combination of the PEEC method with metamodelling results to be very effective in accurately estimating stochastic quantities of interest, while achieving high speed-ups with respect to a MC analysis. Pertinent numerical results have validated the proposed technique.

\section{References}

[1] Ruehli, A. E., Antonini, G., Jiang, L.: 'Circuit Oriented Electromagnetic Modeling Using the PEEC Techniques', (John Wiley \& Sons, Inc., Hoboken, New Jersey, 2017). 
[2] Giselle Fernández-Godino, M., Park, C., Kim N.-H., et al.: 'Review of multi-fidelity models', arXiv:1609.07196, 2016, pp. 1-46.

[3] Koziel, S., Bandler, J. W.: 'Reliable Microwave Modeling by Means of Variable-Fidelity Response Features', IEEE Transactions on Microwave Theory and Techniques, 2015, 18, (12), pp. 4247-4254, doi: 10.1109/TMTT.2015.249526

[4] Giles, M.: 'Multilevel Monte Carlo methods", Acta Numerica, 2015, 24, pp. 259-328, doi:10.1017/S096249291500001X

[5] Dick, J., Kuo, F., Sloan, I.: 'High-dimensional integration: The quasi-Monte Carlo way', Acta Numerica, 2013, 22, pp. 133-288, doi:10.1017/S0962492913000044

[6] Shen, J., Yang, H., Chen, J.: 'Stochastic analysis of variations of electromagnetic properties of composite mixtures', Electronics Letters, 2010, 46, (2), pp. 127-129, doi: 10.1049/el.2010.2932.

[7] Galetzka, A., Bontinck, Z., Römer, U., et al.: 'A Multilevel Monte Carlo Method for High-Dimensional Uncertainty Quantification of Low-Frequency Electromagnetic Devices," IEEE Transactions on Magnetics, 2019, 55, (8), pp. 1-12, doi: 10.1109/TMAG.2019.2911053.

[8] Koziel, S., Leifsson, L., Yang, X-S.: 'Simulation-Driven Modeling and Optimization', (Springer-Verlag, New York, 2014)

[9] Van Nechel, E., Ferranti F., Rolain Y., et al.: 'Model-Driven Design of Microwave Filters Based on Scalable Circuit Models," IEEE Transactions on Microwave Theory and Techniques, 2018, 66, (10), pp. 4390-4396, doi: 10.1109/TMTT.2018.2842197.

[10] Rose, C., Smith, M.D.: 'mathStatica: Mathematical Statistics with Mathematica', (Physica, Heidelberg, 2002)

[11] Pisaroni, M.: 'Multi Level Monte Carlo Methods for Uncertainty Quantification and Robust Design Optimization in Aerodynamics', 2017, PhD thesis, EPFL, Lausanne, Switzerland. 